\title{
Structural basis of anti-PD-L1 monoclonal antibody avelumab for tumor therapy
}

Cell Research (2017) 27:151-153. doi:10.1038/cr.2016.102; published online 30 August 2016

\section{Dear Editor,}

Monoclonal antibodies (mAbs) blocking immune checkpoint molecules, especially programmed cell death 1 (PD-1) and its ligands programmed cell death 1 ligand 1 (PD-L1) and ligand 2 (PD-L2), are currently been investigated for treatment of various tumors [1-3]. PD-L1 and PD-L2 are usually upregulated on the surface of multiple tumor cells to mediate immune tolerance through the interaction with inhibitory PD-1 molecule [4]. Thus, blocking PD-1/PD-Ls interaction has brought promising future for tumor immunotherapy. To date, several PD-1/ PD-L1 blockade antibodies have been approved for clinical use or under phase III clinical trials (e.g., nivolumab, pembrolizumab, atezolizumab, avelumab, durvalumab and BMS-936559, etc.) [4]. The PD-1 targeting therapeutic antibodies block the PD-1/PD-L1 or PD-1/PD$\mathrm{L} 2$ interaction to restore tumor-specific $\mathrm{T}$ cell reactivity, without mediating antibody-dependent cell-mediated cytotoxicity (ADCC). Recently, the structural basis of hPD-1/pembrolizumab (a PD-1 targeting therapeutic antibody developed by Merck \& Co., Inc., USA) has been revealed, providing a molecular insight into blocking PD-1-mediated immune suppression by antibody [5]. PD-L1 targeting therapeutic antibodies possess PD-1/ PD-L1 blockade activity with or without ADCC activity. As one of the PD-L1 targeting antibodies, avelumab is a human IgG1 antibody with ADCC activity developed by Merck (Darmstadt, Germany) and Pfizer, which is now in multiple phase III clinical trials against non-small cell lung cancer (NCT02395172), advanced renal cell cancer (NCT02684006) and gastric cancer (NCT02625610) [6].

The crystal structures of PD-L1 couplexed with its receptor PD-1 have been extensively studied, including human PD-L1 (hPD-L1) alone, mouse PD-1 (mPD-1) complexed with hPD-L1 and human PD-1 (hPD-1) complexed with hPD-L1 [7-9]. Though the complex structure of hPD-1 with a commercial mAb pembrolizumab has been solved very recently [5], hPD-L1/mAb complex structure has not been investigated.

In this study, we expressed the single chain Fv frag- ment ( $\mathrm{scFv}$ ) of avelumab and hPD-L1 with two immunoglobulin (Ig) domains as inclusion bodies in E. coli. Then we applied in vitro refolding method to obtain soluble proteins, and the two refolded proteins can survive well in gel filtration (Supplementary information, Figure S1A). The binding kinetics of avelumab-scFv/hPD-L1 was analyzed by surface plasmon resonance (SPR). The binding avidity was determined by calculating dissociation constant $\left(\mathrm{K}_{\mathrm{d}}\right)$ which was $42.1 \mathrm{pM}$ for avelumab-scFv (Supplementary information, Figure S1B). Subsequently, we performed crystal screen with the avelumab-scFv/ hPD-L1 complex protein, and obtained well-diffractable crystals in $0.2 \mathrm{M}$ magnesium chloride hexahydrate, 0.1 M HEPES-Na, $\mathrm{pH} 7.5,30 \% \mathrm{v} / \mathrm{v}$ isopropanol (see more details in Supplementary information, Data S1).

The crystal structure of the hPD-Ll complexed with avelumab $\mathrm{scFv}$ was determined by molecular replacement at a resolution of $3.2 \AA$ (Supplementary information, Table S1A). The PD-L1 consists of two Ig domains, the $\mathrm{N}$-terminal $\mathrm{IgV}$ domain and the $\mathrm{C}$-terminal $\mathrm{IgC}$ domain. The overall complex structure reveals that avelumab utilizes both heavy chain $\left(\mathrm{V}_{\mathrm{H}}\right)$ and light chain $\left(\mathrm{V}_{\mathrm{L}}\right)$ to bind to the $\mathrm{IgV}$ domain of PD-L1 on the side (Figure 1A and Supplementary information, Figure S1C). The interaction with hPD-L1 involves five of the six complementarity-determining regions (CDRs) of both $\mathrm{V}_{\mathrm{H}}$ and $\mathrm{V}_{\mathrm{L}}$ with a buried area of $\sim 1856 \AA^{2}$. The $V_{H}$ of avelumab dominates the binding to hPD-L1 by all three CDR loops, and $\mathrm{V}_{\mathrm{L}}$ contributes partial contacts by CDR1 and CDR3 loop (Figure 1B and 1C, Supplementary information, Table $\mathrm{S} 1 \mathrm{~B})$, leaving $\mathrm{V}_{\mathrm{L}} \mathrm{CDR} 2$ without binding to hPD-L1. The avelumab-binding epitope region on hPD-L1 is predominantly constituted by the $\mathrm{C}$ strand, $\mathrm{C}^{\prime}$ strand, $\mathrm{F}$ strand, $\mathrm{G}$ strand and $\mathrm{CC}^{\prime}$ loop of hPD-L1 (Figure 1D and Supplementary information, Figure S1C). Notably, the $\mathrm{CC}^{\prime}$ loop of hPD-L1 interacts with CDR3 loops from both $\mathrm{V}_{\mathrm{H}}$ and $\mathrm{V}_{\mathrm{L}}$ of avelumab, involving multiple hydrogen bond interactions. Especially, the residue D61 contributes the major contacts of the $\mathrm{CC}^{\prime}$ loop, including 1 hydrogen bond with residue R99 from LCDR3 loop and 4 hydrogen bonds with residues V104, T105 and T106 from HCDR3 loop 
A
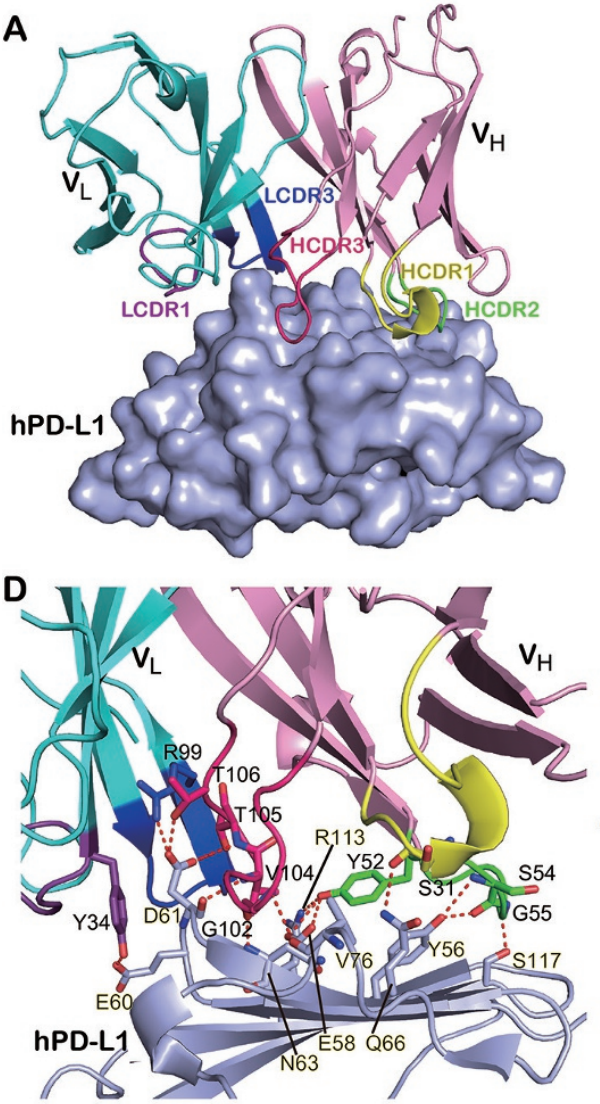

B

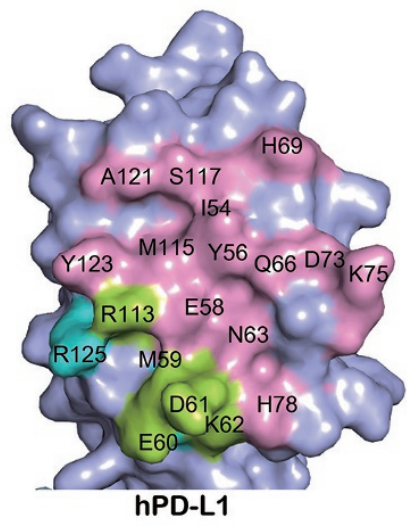

E

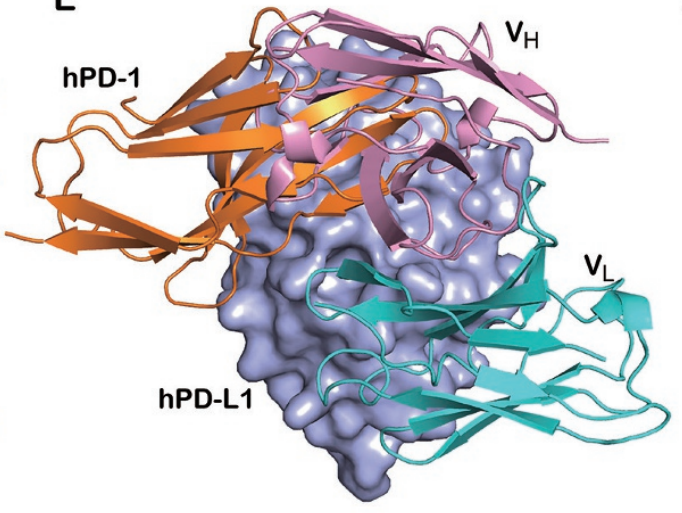

C

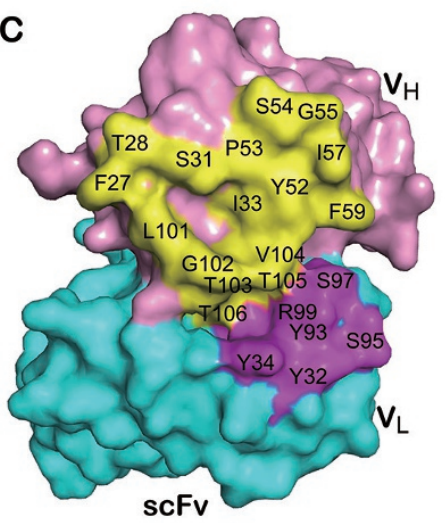

$\mathbf{F}$

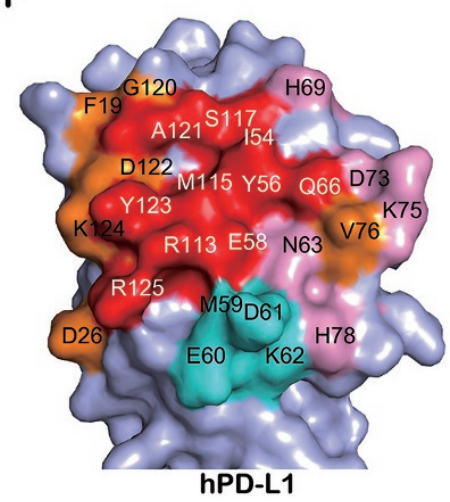

Figure 1 Detailed binding of avelumab to hPD-L1 for the blockade of hPD-1/hPD-L1 interaction. (A) Overall structure of the hPD-L1/avelumab-scFv complex. hPD-L1 (only the mAb-binding IgV domain is depicted) is shown as surface diagram in light blue, and the heavy $\left(\mathrm{V}_{\mathrm{H}}\right)$ and light $\left(\mathrm{V}_{\mathrm{L}}\right)$ chains of scFv are shown as cartoon representations in pink and cyan, respectively. The CDR1, CDR2 and CDR3 loops of $V_{H}$ are colored in yellow, green and hot pink, respectively. The CDR1 and CDR3 loops of $V_{L}$ are colored in purple and blue, respectively. (B) The epitope residues in hPD-L1 are denoted in black characters. Residues contacted by the avelumab-scFV $\mathrm{V}_{\mathrm{H}}$ or $\mathrm{V}_{\mathrm{L}}$ are colored in pink and cyan, respectively, whereas residues contacted by both chains are colored in lemon. (C) The residues of avelumab-scFv contacting hPD-L1 are colored in yellow for $V_{H}$ and colored in magenta for $V_{L}$. (D) The detailed interactions in hPD-L1/avelumab-scFv complex. Residues involved in the hydrogen bond interaction are shown as sticks and labeled. Hydrogen bonds are shown as dash lines. (E) Superposition of the hPD-L1/ avelumab-scFv complex structure with hPD-1/hPD-L1 complex structure. hPD-1 is shown in orange and avelumab-scFv $\mathrm{V}_{\mathrm{H}}$ in pink, $V_{L}$ in cyan, respectively. (F) Binding surface of $h P D-L 1$ by $h P D-1$ or avelumab. The binding residues on $h P D-L 1$ are colored in orange, whereas residues contacted by the avelumab-scFv $\mathrm{V}_{\mathrm{H}}$ or $\mathrm{V}_{\mathrm{L}}$ are colored in pink or cyan, respectively, and the overlapping residues bound by both the receptor hPD-1 and avelumab are colored in red.

(Figure 1D). The buried surface of the four strands (C, $\left.\mathrm{C}^{\prime}, \mathrm{F}, \mathrm{G}\right)$ of hPD-L1 is mainly occupied by HCDR2 and HCDR3 loops (Figure 1C and Supplementary information, Table S1B). The binding involves hydrogen bond interactions between residues (Y52, S54, G55 of HCDR2 and G102 and V104 of HCDR3) of the scFv and residues (Y56, E58, N63, V76, R113 and S117) of hPD-L1. Taken together, the complex structure revealed a $\mathrm{V}_{\mathrm{H}}$-dominated binding pattern between avelumab and hPD-L1.

The structural basis of the anti-PD-L1 antibody blockade was further analyzed by structural superposition of the hPD-1/hPD-Ll complex (PDB: 4ZQK) and the
avelumab-scFv/hPD-L1 complex. The avelumab-binding epitope region on hPD-L1 overlapped with the hPD1-binding region, suggesting that the binding of avelumab with hPD-L1 can impede the binding of hPD-1 to hPD-L1 (Figure 1E). The blockade by avelumab binding is mainly contributed by $\mathrm{V}_{\mathrm{H}}$ with minor contribution from $\mathrm{V}_{\mathrm{L}}$. The detailed analysis of buried surface on hPD-L1 reveals that the overlapping binding area of avelumab and hPD-1 mainly locates on F and G strands which predominantly interact with the HCDR2 loop of the avelumab (Figure 1F and Supplementary information, Figure S1C). The hPD-L1 residues (I54, Y56, M115 and Y123), 
which are critical for forming the hydrophobic core to interact with hPD-1, are occupied by the similar hydrophobic aromatic residues (Y52-G55, I57 and F59) from HCDR2, through interactions including multiple hydrogen bonds [9]. The residues (E58, Q66, R113 and A121) of hPD-L1, which contribute to the binding of hPD-1, also interact predominantly with residues (Y52, P53, I57 and F59) of avelumab. These results indicate that the blockade mechanism of avelumab is that the protruding HCDR2 loop dominates the hPD1-binding region and competes for the binding to hPD-L1.

It is speculated that three competitive binding patterns of the antibody would lead to the blockade of paired molecules to bind each other, i.e., neighboring non-overlapping binding (stereo-specific blockade), partially overlapping binding and completely overlapping binding (e.g., full occupation of high-affinity PD-1 to block PD-1/PDL1 interaction [10]). The blocking mechanism of the avelumab belongs to the partially overlapping pattern, and we showed that the F and G strands of hPD-L1 could be a vulnerable antigenic site for anti-PD-L1 therapeutic antibody. Our findings would benefit the design and optimization of therapeutic antibodies targeting hPD-L1. In the future, whether this partial binding occupation mechanism could be generalized to other anti-PD-L1 therapeutic antibodies or whether there are other blockade hot spots on hPD-L1 yet need further investigation.

The crystal structure of avelumab-scFv in complex with hPD-L1 has been deposited in PDB under accession code: 5GRJ.

\section{Acknowledgments}

This work was supported by the Ministry of Science and Technology of China (973 program: 2013CB531502 and 2014CB542503), the National Natural Science Foundation of China (NSFC; 31390432 and 31500722), Grand S\&T project of China Health and Family Planning Commission (2013ZX10004608-002 and 2016ZX10004201-009), the Strategic Priority Research Program of the Chinese Academy of Sciences (CAS; XDB08020100). We thank the staff of BL17U beamline at Shanghai Synchrotron Radiation Facility for assistance with data collection. YS is sup- ported by the Excellent Young Scientist Program of CAS and the Youth Innovation Promotion Association of CAS (2015078). GFG is supported partly as a leading principal investigator of the NSFC Innovative Research Group (81321063).

$$
\begin{aligned}
& \text { Kefang Liu }{ }^{1,2, *} \text {, Shuguang Tan }{ }^{3, *} \text {, Yan Chai }{ }^{3} \text {, } \\
& \text { Danqing Chen }{ }^{3} \text {, Hao Song }{ }^{4} \text {, } \\
& \text { Catherine Wei-Hong Zhang, Yi Shi }{ }^{3} \text {, Jun Liu }{ }^{1,2} \text {, }
\end{aligned}
$$$$
\text { Wenjie Tan }{ }^{1,2} \text {, Jianxin Lyu }{ }^{1} \text {, Shan Gao }{ }^{6} \text {, Jinghua } \text { Yan }^{7} \text {, }
$$$$
\text { Jianxun } \mathrm{Qi}^{3} \text {, George F Gao }{ }^{1,2,3}
$$

${ }^{I}$ College of Laboratory Medicine and Life Sciences, Wenzhou Medical University, Wenzhou, Zhejiang 325035, China, ${ }^{2}$ National Institute for Viral Disease Control and Prevention, Chinese Center for Disease Control and Prevention (China CDC), Beijing 102206, China; ${ }^{3}$ CAS Key Laboratory of Pathogenic Microbiology and Immunology, Institute of Microbiology, Chinese Academy of Sciences, Beijing 100101, China; ${ }^{4}$ Research Network of Immunity and Health (RNIH), Beijing Institutes of Life Science, Chinese Academy of Sciences, Beijing 100101, China; ${ }^{5}$ ImmuFucell Biotechnology Co., Ltd., Beijing 100102, China; ${ }^{6}$ CAS Key Laboratory of Bio-medical Diagnostics, Suzhou Institute of Biomedical Engineering and Technology, Chinese Academy of Sciences, Suzhou, Jiangsu 215163, China; ${ }^{7}$ CAS Key Laboratory of Microbial Physiological and Metabolic Engineering, Institute of Microbiology, Chinese Academy of Sciences, Beijing 100101, China *These two authors contributed equally to this work.

Correspondence: George F Gao ${ }^{\mathrm{a}}$, Jianxun $\mathrm{Qi}^{\mathrm{b}}$

${ }^{\mathrm{a}} \mathrm{Tel}:+861064807688$

E-mail: gaof@im.ac.cn

${ }^{\mathrm{b}} \mathrm{Tel}:+861064807417$

E-mail: jxqi@im.ac.cn

\section{References}

1 Powles T, Eder JP, Fine GD, et al. Nature 2014; 515:558.

2 Rosenberg JE, Hoffman-Censits J, Powles T, et al. Lancet 2016; 387:1909-1920.

3 Tan S, Gao GF. Chinese Sci Bull 2015; 60:3155-3157. (In Chinese)

4 Callahan MK, Postow MA, Wolchok JD. Immunity 2016; 44:10691078.

5 Na Z, Yeo SP, Bharath SR, et al. Cell Res 2017; 27:147-150.

6 Madan RA, Donahue RN, Singh H, et al. J Clin Oncol 2016; 34(suppl 2S):a214.

7 Chen Y, Liu P, Gao F, et al. Protein Cell 2010; 1:153-160.

8 Lin DY, Tanaka Y, Iwasaki M, et al. Proc Natl Acad Sci USA 2008; 105:3011-3016.

9 Zak KM, Kitel R, Przetocka S, et al. Structure 2015; 23:2341-2348.

10 Maute RL, Gordon SR, Mayer AT, et al. Proc Natl Acad Sci USA 2015; 112:E6506-E6514.

(Supplementary information is linked to the online version of the paper on the Cell Research website.) 